\title{
Exploring Mathematical Models to Improve Routing Protocol for Call Centre
}

\author{
Ese Sophia Mughele \\ Delta State School of Marine Technology \\ Burutu, Nigeria. Department of Computer \\ Science, School of Science \& Technology
}

\author{
Konyeha Susan \\ University of Benin, Benin City, Nigeria, \\ Department of Computer Science, \\ Faculty of Physical Science
}

\begin{abstract}
Customers usually experience queue often times when they call a call center to meet their information need or make an inquiry. The queue experienced by customers at call centres can be alarming often times. Many customers are irritated by the long time spent on the queue before their calls are been answered. Call center agents are trained to handle all entry calls to a call centre but are they characterized with different performance level for the call in terms of average call handling time (AHT) and call resolution (CR). The main purpose of this paper is to evaluate the performance of hybridized routing rule Hybrid Heterogeneous call Routing Rule (HHCRR) proposed by (Mughele et al, 2017b). The method adopted is mathematical techniques by assuming 15 working hours of the day and subjected the period of time assumed into the equation. Since the proposed hybrid rule is designed to optimize, the study determined the viability of the rule by testing the hybrid rule mathematically. The result from the mathematical techniques deployed shows minimization of wait-time and maximization of call resolution hence optimization is achieved, validating the hybrid rule to be implementable.
\end{abstract}

\section{Keywords}

Routing Rule, Hybrid Rule, Call Center, Mathematical Techniques

\section{INTRODUCTION}

Call center is a division within an organisation and it focuses on inbound and outbound communication with customers. The main goal for the call center management, especially in a governing body, is to ensure customer satisfaction. A customer during a service encounter experiences either one or both of the followings namely: the time spent waiting for the service and the service itself. Call centres give priority to the two criteria with emphasis on one more than the other. Those that place more emphasis on time spent waiting for the service are more concerned with reducing the average time involved in handling a call while those that are concerned with the service itself aims at effective resolution of customer issues

Armony (2005) says for a call centre to reduce waiting lines with emphasis on the reduction of time spent, its best to route calls to agents who can handle customer issues the fastest, sometimes even holding a call in queue to wait for that agent than routing the call to a slower agent. This might lead to further increase in congestion, repeat calls from unreceptive issues and undue burden on some agents.

Vericourt et al. (2005), states that for a call centre to reduce waiting lines, emphasis should be on the service itself that is; call resolution. Its best to route calls to agents who resolve customer issues, sometimes holding a call in queue to wait for such agent this might also lead to increase in congestion and undue burden on some agents. After a customer has received service from a call centre agent on a particular issue, a subsequent call from that customer about the same issue is a clear sign that the issue had not been resolved during the previous service encounter, and this lack of resolution is a strong sign of customer dissatisfaction. Call center agents are trained to handle all entry calls to a call centre but they are characterized with different performance level for the call in terms of average call handling time (AHT) and call resolution (CR). .Hence there is need to hybridize both wait-time and call resolution routing rule to enhance performance. The main purpose of this paper is to mathematically demonstrate that the hybrid rule/algorithm is effective and can be implemented to obtain optimality when deployed for call center operation.

\section{RELATED LITERATURE}

Customer service call centres have obviously become a very integral part of many organisations' business operations today, inbound call centres employ millions of agents across the globe and serve as a primary customer-facing channel for many different industries. There has also been a great deal of research interest in call centre operations management, with the extensive and evolving literature thoroughly analysed (Mehrotra et al, 2009). Hart et al. (2006) provides a complete review of articles on First Call Resolution (FCR), while also pointing out the importance of measuring and using FCR. Resolving customer queries the first time around is a commonly shared goal.

Zhan and Ward (2006) noted that the challenge in call centre operation is how to determine the relevant control in the routing; that is, the decision concerning which agent should handle an arriving call when more than one agent is available. Garcia. et al. (2012), noted that as time spent on queue at the call centres increases, it becomes unacceptable for customers, and this affect their satisfaction level.

Stanley et al (2008) posited that in a service base call centre, the two key challenges are (i. Where should a call be routed to and) (ii. Who should handle the call?) They deployed base case FIFO approach for the simulation to analyse performance-based routing strategies in call centres. Their work shows the potential for significant improvements in call centre performance especially Average Speed to Answer (ASA). This was achieved by using rules based on historic performance data such as Average call Handling Time (AHT) and first call Resolution (FCR) rates.

Garcia. et al. (2012), noted that as time spent on queue at the call centres increases, it becomes unacceptable for customers, and this affect their satisfaction level. A study was conducted using Univariate Analysis of Variance (ANOVA) to determine customer's perception of their wait 
experience at call centres. Their result showed that though the time spent on the queue waiting can lead to customer's dissatisfaction, nevertheless, it is not as important as the agent's ability. More so, the concept of routing rules to be deployed for efficient call resolution rate was not emphasised. One important measure is the Average Handling Time (AHT) which depicts the productivity or the time it takes for the agent to handle the calls on average. The other is the Call Resolution (CR) that measures the number of times an inquiry by a caller is completed effectively the first time without the need for a follow-up or the second call from the customer (Brantevica, 2018)

Dabrowski (2013) observed that the key performance indicators to measure call centre metrics performance such as average speed of answer, cost per call, agent utilization rate, first contract resolution rate, customer satisfaction and aggregate call centre performance are not effectively maximised. He used CallLogic system to improve the fundamental call routing logic of the Northeast Utilities call centres. Although the findings from the CallLogic system led to discoveries and ideas on how to improve the fundamental call routing logic of the Northeast Utilities call centres, the CallLogic project achieved high success in the average call handling time. The study only made mention of call Resolution rate and its impact on operational success.

The quality of service accessibility and customer waiting time are dominant performance measures (Vericourt and Zhou 2005b).

Jouine et al (2007), considered two basic multiclass call center models, with and without reneging the challenge / problem is that customers at times encounter delay upon arrival at a call center. The first method used was to estimate virtual delays that will be used within the announcement step. Their second model, they develop a call center incorporating reneging, The model takes into consideration change in customer behavior that may occur when delay signals or information is communicated to the customers in order to estimate virtual delays of new arrivals. To improve customers satisfaction and reduce congestion, call center have in recent times started experimenting by informing arriving customers about anticipated delay, this is noted in (Armony and Maglaras 2004), When customers are waiting on the queue, they have no means of estimating the queue lengths or progress rate, hence there is tendency for increase in axiety during the waiting period. Basically, Jouine et al (2007) from the result presented were able to model customer's reactions to delay announcements in such a setting with priorities and to provide an analysis for this case. The study also introduces the need for announcing delays when a new customer finds the queue empty.

Adan et al. (2013), in a related work, considered a system based on assumption that the system is overloaded and a such all server are always busy and a fraction of the customers are forced to abandonment. They deployed FCFS and skilled based routing rule methodology. The authors also emphasized that to optimize performance, determining the right level of cross-training seems more important than laying more emphasis on the choice of routing rule.

Adan et al, (2013), specifies the system as follows;

customers are of types $\mathrm{C}=\{\mathrm{C} 1-\cdots, \mathrm{C} 1\}$, servers are of types $\mathrm{S}=\{\mathrm{S} 1$ - - - -,SJ $\}$, and a bipartite gragh $\mathrm{G}$ of compatible matches between C,S. Graph G specifies the level of cross-training; it has arc $(i, j) £ G$ if server type $S j$ has the skills to serve the customer type Ci. An additional assumption follows:

Customers of type $\mathrm{Ci}$ arrive in independent Poisson stream or distribution of rate $\lambda_{i}$, and have patience distribution $F_{i}$ which is absolutely continuous. There exist $\Pi \hat{J}$ servers of type $\dot{C}_{i}$ by a server of type $\mathrm{Sj}$ has a random duration distributed as Gij, with rate $\mu_{i j}=1 / \mu_{i} j$. The notation subscript $I$ for customers of type $\mathrm{Ci}$ and subscript $\mathrm{j}$ for servers of type $\mathrm{Sj}$. Given that data $\lambda i, F i, G, n j$, Gij, under FCFS policy, include waiting times abandonment rates, routing flows between customer types and server types, and workload of each server type.

Selvi and Sathya (2012) observed that the Erlang B model is a formular for blocking, a probability derived from Erlang distribution. The Erlang B describes an unsuccessful call, when all servers are busy and the call is neither queued nor retired but loss completely. It is assumed that calls attempts arrive following a passion process (Selvi and Sathy, 2012; Osahenvem and Odiase 2016 and Aldor-Norman et al, 2010), so calls are independent. More also, it is assumed that message length (holding times) are exponentially distributed as depicted in (Markovian system) and this is generally applied under general holding time distributions. Usually, Erlangs are dimensionless quantities as average call arrival rate $\lambda$, which is multiplied by average call length, $h$. Blocking occurs when there are new calls waiting to be served and all servers are already busy. The Erlang B formular assumes that blocked traffic is immediately cleared.

Dabrowski (2013) observed that the key performance indicators to measure call centre metrics performance such as average speed of answer, cost per call, agent utilization rate. Mehrotra et al (2012), developed a framework for the optimisation of routing rules using first-come-first serve (FCFS) among wait-time routing rule and Probability Routing (PR), among call resolution routing rule to develop a hybrid rule

Mughele and Chiemeke (2016) evaluated the three call resolution oriented routing rules discussed by Mehrotra et al 2012. A similar study was conducted by Mughele et al (2017a) where a comparative analysis of waiting time routing rules for queue reduction in call center. Mughele et al, $(2017 \mathrm{c})$, deployed a simulation model that can be used for the optimisation of a call center. They deployed discrete event driven simulation to optimise the routing rule among call resolution orienting rule which is indicated to be SOR (Mughele and Chiemeke 2016).. In a related study conducted by (Mughele and chiemeke 2017), the authors specifically addressed the problem associated with the utilization of call center system in terms of service rate, waiting time of agent in an agent group. The number of call type $i$ handled by a call center is determined by the maximal service rate of the agents in agent group $\mathrm{j}$. The techniques they deployed was graph theory analysis to enhance optimization

Brantevica (2018) establish the need for a tradeoff between the two performance metrics of AHT and FCR depending on the need and the operation the call center based on the decision if the call center manager. AHT and FCR are importance performance metrics of call centre therefore; models and frameworks should be developed to integrate the algorithm of both metrics in the bid to improve routing protocol. Some researchers have developed hybrid models and frameworks that actually integrated both wait-time and call resolution routing rules which enhances $\mathrm{CR}$ and also 
reduce wait-time (Mehrotra et al 2012 and Mughele et al, 2017b).

Mughele et al (2017b) developed a framework as a single routing rule that is able to solve Min/Max problem simultaneously in call center. This paper is an extension of (Mughele et al 2017b). This study is set to determine if the hybrid rule (HHCRR) proposed by Mughele et al (2017b), is efficient and implementable by mathematically evaluating the hybrid rule to determine its efficiency.

\section{METHODOLOGY}

This study basically, set out to evaluate the hybrid model, a framework developed by Mughele et al, 2017b, by deploying mathematical techniques. Previous studies conducted, compared the algorithms of some existing routing rules to determine the optimal. Four of the routing rules were waittime routing rules and three were call resolution routing rule as obtained from (Mehrotra et al, 2012; Mughele and Chiemeke, 2016; Mughele et al 2017a). The algorithms were tested with date collected from a Call Center of a Telecommunication Organisation in Nigeria. The optimal routing rule for wait-time and call resolution routing rules were SSTF and SQR respectively (Mughele and chiemeke, 2017). The algorithm for the two routing rules were hybridised, and tested with data from the call center to determine its performance, the hybrid was called Hybrid Heterogeneous Call Routing (CR) Rule (HHCRR) (Mughele et al 2017b). The performance of the algorithm was determined mathematically by dry running using the equations for MIN/MAX. The mathematical procedure adopted was dry running of the hybrid algorithm by assuming 15 working hours of the day,

As adapted from Mehrotra et al. (2012), the benchmark routing rule will be the First-Come-First-Served/LongestWait (FCFS/LW) rule, because this is the routing rule deployed in majority of the call centres. The following model consist of the algorithm for the hybrid routing rule, which is made up the optimal of the wait-time routing rule (SSTF) (Mughele and Chiemeke 2016) and CR rate routing rule (SQR) (Mughele et al 2017a).

Table 1 contains the operational variables deployed for the equations in this study.

Table 1: Operationalisation of research variables

\begin{tabular}{|c|c|}
\hline Variable & Description of variables \\
\hline time periods $t_{i}$ & $\begin{array}{c}\text { time period per day }: 7 \text { am to 9pm for all } \\
\text { agents that is } 15 \text { hours per day }\end{array}$ \\
\hline Call type $\mathrm{i}$ & $\begin{array}{c}\text { Multiple call types such that } \mathrm{i}=1,2 \ldots \mathrm{I} \\
\text { where } \mathrm{I} \text { is } 8 \text { in our model }\end{array}$ \\
\hline Agent $\mathrm{j}$ & $\begin{array}{c}\text { Multiple agent groups such that } \mathrm{j}=1,2 \\
\text {...J. where } \mathrm{J} \text { is } 35 \text { in our model }\end{array}$ \\
\hline $\mathrm{C}_{\mathrm{j}, \mathrm{t}}$ & $\begin{array}{c}\text { Cost of an agent of type } \mathrm{j} \text { having/working } \\
\text { in time } \mathrm{t}\end{array}$ \\
\hline$p_{i}$ & $\begin{array}{c}\text { represent the proportion of call type } \mathrm{i} \\
\text { from the total new arrival that goes into } \\
\text { the various call type } \mathrm{i} \text { queue }\end{array}$ \\
\hline $\mathrm{Q}_{\mathrm{i}}(\mathrm{t})$ & $\begin{array}{c}\text { number of type } \mathrm{i} \text { call waiting for service } \\
\text { at time } \mathrm{t}\end{array}$ \\
\hline $\mathrm{f}_{\mathrm{j}}(\mathrm{t})$ & $\begin{array}{c}\text { number of available agents of group } \mathrm{j} \\
\text { who are free at time } \mathrm{t} \text {, where } 0 \leq \mathrm{f}_{\mathrm{j}}(\mathrm{t}) \leq \mathrm{n}_{\mathrm{j}},\end{array}$ \\
\hline
\end{tabular}

\begin{tabular}{|c|c|}
\hline & for all $\mathrm{j}, \mathrm{t}$. \\
\hline$\lambda_{i}$ & arrive rate of calls of type $i$ \\
\hline$\lambda_{T}$ & The total arrival rate \\
\hline $\mathrm{n}_{\mathrm{j}}$ & no of agents in group $j$, such that $n_{j} \varepsilon Z^{+}$ \\
\hline$\overline{X_{i j}}$ & $\begin{array}{l}\text { proportion of calls type i routed to agent } \\
\text { group } \mathrm{j}\end{array}$ \\
\hline $\mathrm{X}_{\mathrm{ij}, \mathrm{t}}$ & $\begin{array}{l}\text { proportion of calls type i routed to agent } \\
\text { group } \mathrm{j} \text { at time } \mathrm{t}\end{array}$ \\
\hline $\mathrm{y}_{\mathrm{ij}, \mathrm{t}}$ & $\begin{array}{l}\text { No of agents in agent group } \mathrm{j} \text { that handles } \\
\text { call type } \mathrm{i} \text { at time } \mathrm{t}\end{array}$ \\
\hline$\mu_{\mathrm{ij}}$ & $\begin{array}{l}\text { service rate of Agent group } \mathrm{j} \text { for call of } \\
\text { type } \mathrm{i}\end{array}$ \\
\hline$\mu_{z}$ & $\begin{array}{c}\text { service rate of Agent group } \mathrm{j} \text { for call of } \\
\text { type } \mathrm{i}\end{array}$ \\
\hline$\beta_{i}$ & $\begin{array}{l}\text { arrival of unresolved calls of call type i } \\
\text { who call back }\end{array}$ \\
\hline$\beta_{i j}$ & $\begin{array}{l}\text { total arrival rate of agent group } \mathrm{j} \text { for call } \\
\text { type i who call back. }\end{array}$ \\
\hline$\theta_{i j}$ & $\begin{array}{l}\text { resolution probability of agent group } \mathrm{j} \text { of } \\
\text { call type } \mathrm{i}\end{array}$ \\
\hline$\rho_{j}$ & total utilization of agent group $\mathrm{j}$ \\
\hline$\Gamma_{i-} \Gamma_{i+}$ & $\begin{array}{l}\text { the lower and upper bound such that } \\
\text { each call type i must be served at total } \\
\text { utilization between bounds }\end{array}$ \\
\hline$\rho$ & proportion of time each server is busy \\
\hline
\end{tabular}

\subsection{Proposed Hybrid Routing Rule}

The focus here is on a rule that combines the optimal rule for call resolution and waiting time routing rules. As identified in previous studies, Shortest Service Time First (SSTF) was the most optimal for Waiting-Time Routing Rules and Shortest Queue Routing (SQR) was the most optimal for Resolution Probabilistic Routing Rules.

The hybrid algorithm, the following are assumed

1. A call of a particular type that arrives when agents of multiple matching groups are free will be routed to a matching agent group (j) that has the relatives Shortest Service Time and shortest queue for that call type.

2. Let $t_{i}$ represent the time period per day $7 \mathrm{am}$ to $9 \mathrm{pm}$ i.e. 15 hours per day

3. Let Multiple call types be indexed by $\mathrm{i}=1,2 \ldots \mathrm{I}$ where I is 8 in our model

4. Let Multiple agent groups be indexed by $\mathrm{j}=1,2$ ...J. where $\mathrm{J}$ is 35 in our model

5. Let $p_{i}$ represent the proportion of call type $\mathrm{i}$ from the total new arrival that goes into the various call type i queue. This is computed using

$p_{i}$

$$
=\frac{\text { Total Calls of type } i}{\text { total Calls }}
$$


6. Let $\mathrm{Q}_{\mathrm{i}}(\mathrm{t})$ represents the number of type $\mathrm{i}$ call waiting for service at time $t$

7. Let $f_{j}(t)$ be the number of available agents of group $\mathrm{j}$ who are free at time $t$, where $0 \leq \mathrm{f}_{\mathrm{j}}(\mathrm{t}) \leq \mathrm{n}_{\mathrm{j}}$, for all $\mathrm{j}$, t.

8. Let $\lambda_{i}$. represents arrival rate of calls of type i such that $\lambda_{i}=t_{i} \lambda$

9. Let $n_{j}$ represent no of agents in group $j$, with $n_{j} \varepsilon$ $\mathrm{Z}^{+}$

10. Let Xij represent proportion of calls type i routed to agent group $\mathrm{j}$

11. Let $\mu_{\mathrm{ij}}$ represent service rate of Agent group $\mathrm{j}$ for call of type $i$. This is computed by

$u_{i j}$

$=\frac{1}{\text { AverateHandlingTime }_{j}} \ldots \ldots \ldots \ldots \ldots$ Equation 2

12. Let $\mu_{z}$ represents the total service rate of call type $\mathrm{i}$ computed using equation

$\mu_{z}$

$=\sum_{j=1}^{j} n_{j} u_{i j}\left(\frac{\frac{\lambda_{i j}}{u_{i j}}}{\sum_{i^{\prime}} \frac{\lambda_{i^{\prime} j}}{u_{i^{\prime} j}}}\right) \ldots \ldots \ldots \ldots \ldots \ldots$ Equation 3

13. Let $\beta_{i}$ accounts for arrival of unresolved calls of call type i who call back. This is computed using equation

$$
\begin{aligned}
& \beta_{i} \\
& =\frac{\lambda_{i}}{1-\sum_{j}(1-\theta i j) x_{i j}} \ldots \ldots \ldots \ldots \ldots . . . . . . . \text { Equation } 4
\end{aligned}
$$

14. Let $\beta_{i j}$ represents total rate of available agent group $\mathrm{j}$ for call type $\mathrm{i}$ who call back.

$$
\beta_{i j}=\frac{\beta_{i} x_{i j}}{n_{j}} \ldots \ldots \ldots \ldots \ldots \ldots \ldots \ldots \ldots \ldots \ldots \text { Equation } 5
$$

15. Let $\theta_{i j}$ represent the resolution probability of agent group $\mathrm{j}$ of call type $\mathrm{i}$. This is the proportion of calls type $\mathrm{i}$ resolved by agent group $\mathrm{j}$ with total arrival rate of $1-\beta_{i}$

16. Let $\rho_{j}$ represent total utilization of agent group $\mathrm{j}$. This is computed by equation

$$
\rho_{j}=\sum_{i} \frac{\beta_{i} x_{i j}}{\mu_{i j}} / n_{j}
$$

Equation 6

17. $\Gamma_{i-}$ and $\Gamma_{i+}$ represent the lower and upper bound such that each call type i must be served at total utilization between bounds.

$\Gamma_{i}=\frac{\beta_{i}}{\mu_{z}}$ Equations 7

\subsection{Mathematical Procedure to Test-Run Hybrid Routing Rule (HHCRR)}

The study assumes a Call Center with 15 hours of working period of the day. Inserting the time into the algorithm, this will enable us optimize mathematically, by reducing time spent on the queue and at the same time enhance resolution rate. $t_{i}=\frac{\text { period }}{\text { day }}($ we assume 15hrs) working hourin a day

$t_{i}=15$

$t_{2}=15+1($ incramental step for each itertion $)$

OR

$t_{i}=15$

then $t_{1}=15$

$t_{2}=t_{1}+1$

Where $i=1,2,3,4,5,6,7,8$

$$
\begin{aligned}
& I=i \\
& \quad t_{i}=15
\end{aligned}
$$

For every customer call, and agent attended to at the allocated daily working hours of 15 hours is illustrated as,

$t_{i}=15 / x$, where $\mathrm{x}$ signify customers time for each call received by the agent.

And $x$ ranges from $x_{1, \ldots \ldots . .,} x_{n}$

$t_{2}=\frac{15}{x_{2}}$, then $x_{2}=15 / t_{2}$

$j=1,2,3,4, \ldots 35$

$\mu i j=1 /$ Average handling time

$\mu_{i x j}=1 / \epsilon j \quad j=35$

$T_{j}=35$ Where $\mathrm{Tj}$ define agents for each call

$T j=35 / y$,

define per call for each respondent from custormer care agent

$t_{2}=35 / y 2$

$\mu_{i x j}=$ Avg $=$ Total call $/$ Total agent

$\rho_{j}=\sum_{i} \frac{\beta_{i} x_{i j}}{\mu_{i j}} / n_{j}$

let $P_{i}=60$

The matrix table 1: consist of data from data set

\begin{tabular}{|c|c|c|c|c|c|c|c|}
\hline$i$ & $i^{i}$ & $j^{\prime}$ & $U_{i j}$ & $X_{i j}$ & $\mu_{j}$ & $i$ & $j$ \\
\hline 1 & 1 & 2 & 3 & 3 & 3 & 1 & 4 \\
\hline 2 & $1 / 2$ & 3 & 6 & 6 & 6 & 3 & 2 \\
\hline 3 & $1 / 3$ & 4 & 12 & 12 & 12 & 2 & 5 \\
\hline$\equiv$ & $\equiv$ & $\equiv$ & $\equiv$ & $\equiv$ & $\equiv$ & 8 & 3 \\
\hline$\gamma$ & $\sum 1 / i$ & 35 & $\sum U_{i j}$ & $\sum X_{i j}$ & $\sum \mu_{i j}$ & & \\
& & & & & & \\
\hline
\end{tabular}


$U_{z}=\left(\sum_{j=1}^{i} \eta_{j} U_{i j}\right)\left(\frac{\frac{\lambda_{i j}}{U_{i j}}}{\sum_{i 1} \frac{\lambda_{i j}}{U_{i j}}}\right)$

$i=1$

$1,2, \ldots .35$

$i=2$

$\sum_{4=1}^{4} \eta_{i U_{i j}}=(2(3)+3(6)+4(12))$

$=6+18+48$

$\sum \eta_{i} U_{i j}=72$

where $\quad i=1, \ldots, 8$

$\lambda_{i}=\operatorname{Li} \lambda$

$\lambda_{i}=1$

$$
\lambda_{1}=\left(15 / x_{1}\right)=15(1)=15
$$

$$
\lambda_{2}=15=2 \times \frac{15}{2}=7.5
$$

This justifies that $\lambda_{i}$ denoted as $\mathrm{i}$ is decreasing in a consistent order

$\left.U_{z}=72\left(\frac{T_{1}=\frac{15}{3 / 3} x_{1}}{3 / 1 / 1 / 3}\right)^{1 / 3}\right)^{x}$

$72\left(\frac{3 / 3}{3 \times 3}\right)=72(1 / 9)$

$U_{z}=72 / 9$

$$
\begin{aligned}
& \beta_{i}=\frac{\lambda_{i}}{1-\sum_{i}(1-\theta) x_{i j}} \\
& \lambda_{i}=t_{1} \lambda=\quad i=1 \ldots, 8
\end{aligned}
$$

$\lambda_{1}=t_{1} \lambda_{1}^{0}$

$\lambda=1$

$\lambda_{1}=\lambda$

$\lambda_{2}=\lambda_{1}$

$\left(t_{2}=t_{1}+1\right)$

$\lambda_{1}=15(1)$

$\lambda_{1}=15$

$\lambda_{2}=t_{2} \lambda_{1}$

$\lambda_{2}=\left(t_{1}+1\right) 15 \mathrm{~min}$

$\lambda_{2}=(15+1)(15)$

$\lambda_{2}=16(15) \ldots \ldots \ldots \lambda_{n}$

$\theta=$ Prepare the resolution rate

$\theta_{i}=$ Resolution of $i I=1,2, \ldots, 8$

$\theta_{i j}=$ Resolution of $\theta_{x j}$

$j=1, \ldots \ldots, 35$

$i=1, \ldots \ldots, 8$

$\theta_{i}=\frac{1}{D}$

Probability of the resolution rate must be less than one (1)

$\lambda_{1}=50$

$\sum(1-t)=0.95$ 


$$
\begin{aligned}
& \beta=\frac{50}{11} \\
& \beta_{i}=\frac{50}{1-(-0.9)} \\
& \frac{50}{1-0.9} \\
& \frac{10-1}{10}=\frac{9}{10}=50 / 9 / 10 \\
& \beta_{1}==50 / 9 / 10
\end{aligned}
$$

Table 2: From matrix table

\begin{tabular}{|c|c|c|c|c|c|}
\hline & $C_{i j}$ & 2 & 3 & 4 & 5 \\
\hline 1 & 1 & 3 & 5 & 2 & 8 \\
\hline 2 & 4 & 5 & 1 & 7 & 6 \\
\hline 3 & 8 & 9 & 1 & 9 & $(10)$ \\
\hline
\end{tabular}

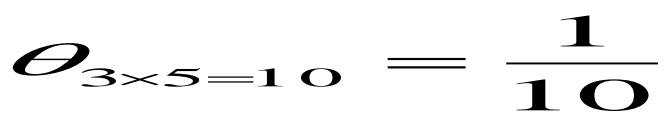

$\beta_{1}=\frac{500}{9} \quad \frac{1}{2}=0.2$

$\beta_{2}=120$

Probability of resolution rate must be less than one

$\theta_{i j}=\frac{1}{3}=0.333$

$\sum_{j}\left(1-\theta_{i j}\right) x_{i j}$

$j=1 \quad$ or $\quad y=35$

Using the parameters from the extracted table 2 above, the following is computed

$\Sigma_{j}\left(1-\theta_{i j}\right) X_{i j}$

$\Sigma_{1}\left(1-\theta_{2 \times 1}\right) X_{2 \times 1} \quad n_{j}=J=$ agent

$\Sigma_{1}(1-0.333)(0.33)$ where $J=1,2, \ldots, 35$

$\beta_{1}=\frac{\lambda_{1}}{1-(1-0.333)(0.33)}=\frac{50}{1-(0.2211)}=\frac{50}{0.7789}$ $\beta_{1}=64.2$

$\beta_{2}=\frac{\lambda_{2}}{1-\Sigma_{3}\left(1-\theta_{i . j}\right) x_{i j}}$

where $\lambda_{2}=\lambda_{1}+1=50+1=51$

$\beta_{2}=\frac{51}{1-\left(1-\theta_{2 \times 3}\right)\left(x_{2 \times 3}\right)}=\frac{51}{1-(1-1)\left(\frac{1}{6}\right)}$

$\beta_{2}=\frac{51}{1-0}=\frac{51}{1}=51$

This justifies that resolution rate is increasing at a consistent orde

$\beta_{8}=\frac{X_{8}}{\left.1-\Sigma\left(1-\theta_{i j}\right) x_{i j}\right)}$

$\beta_{i j}=\frac{\beta_{i} x_{i j}}{n_{i}}=\beta_{1,2}=\frac{642(0.33)}{35}=64.2 \times \frac{0.33}{35}=0.7$

$P_{j}=\frac{\left(\Sigma_{i} \frac{\beta_{i} x_{i j}}{\mu_{i j}}\right)}{n_{j}} i=1, \ldots, 8 ; \quad j=2$

$P_{2}=\frac{\left(\Sigma_{1} \frac{\beta_{1} x_{1,2}}{\mu_{1,2}}\right)}{n_{2}}=\frac{\left(\frac{642}{3}\right)}{12}=10.7$

$P_{2}=10.7$

$r_{i}=\frac{\beta_{i}}{i \mu_{2}}$

$r_{1}=\frac{\beta_{1}}{\mu_{2}}$

$\beta_{1}=64.2$

$\mu_{2}=\frac{72}{9}$

$r_{1}=\frac{64.2}{\frac{72}{9}}=\frac{64.2 \times 9}{72}=8.025$

\section{DISCUSSIONS}

The result from the mathematical techniques shows a consistent reduction of the values of $\mathrm{i}$ from 15 minutes to 7.5minutes this value will continue to reduce as computation is conducted further. This value implies that wait-time of calls in the queue keep reducing consistently. While the resolution rates on the other hand increases consistently, rating from 50 to 51 resolution rate. The simultaneous decrease and increase of the values for wait-time and call resolution validates the fact that optimization is achieved with the hybrid routing rule. The hybrid algorithm can further be implemented and designed to improve and enhance call center operations and also increase customer satisfaction and brand loyalty. The hybrid rule if implemented and deployed will be able to resolve both the challenge of wait-time on queue and effective call resolution by proffering low wait-time and enhanced call resolution rate

\section{CONCLUSION}

The result from the mathematical techniques of the hybrid algorithm shows that optimization was achieved mathematically, by assuming 15 hour working hours of the day. The value obtained from mathematically equation with the assumed values proves that the proposed hybrid routing rule can be designed, developed and implemented. This will 
enhance call center operations, optimization and improves customer satisfaction.

\section{FUTURE RESEARCH}

This paper only considered the performance of the hybrid framework to enhance call handling for call center management by mathematically evaluating and testing the hybrid rule proposed by (Mughele et al, 2017b). Further research can be conducted in the following areas;

i. The role of Customer Relationship Management $(\mathrm{CRM})$ in enhancing the efficiency of call handling in call center operations

ii. Exploring the domain of knowledge based system to increase the efficiency of call center management

\section{ACKNOWLEDGEMENTS}

We are grateful to the Marie Curie Library of the Abdus Salam International Centre for Theoretical Physics (ICTP) for permission to use the eJDS facilities for reference searching and MES appreciate the European Centre for Research Training and Development UK for access to research materials

\section{REFERENCES}

[1] Adan .I, Boon .M and Weiss .G (2013), Design and evaluation of overloaded service systems with skilled based routing, under FCFS policies. Accessed May 2017

[2] Aldor-Noiman S, Feigin D and Mandelbaum A (2010), Workload Forecasting For a Call Center: Methodology and A Case Study. The Annals of Applied Statistics 2009, Vol. 3, No. 4, 1403-1447 DOI: 10.1214/09AOAS255 c (c) Institute of Mathematical Statistics, 2009

[3] Armony M. and Maglaras C. (2004) On Customer Contact Centers with a Call-Back Option: Customer Decisions, Routing Rules, and System Design. OPERATIONS RESEARCH Vol. 52(2) March-April 2004, pp.271-292

[4] Armony M. (2005), Dynamic routing in large-scale service systems with heterogeneous servers. Queueing Systems, 51(3-4):287-329.

[5] Brantevica L. (2018) Investigating the relation between First Call Resolution and Average Handling Time in a call center Research paper Business Analytics. Analytics Vrije Universiteit Amsterdam , Faculty of Science M.sc thesis, Research paper Business. Accessed April 2020.

[6] Dabrowski M. (2013), Business Intelligence In Call Centers. International Journal of Issue Computer and Information Technology (ISSN: 2279 - 0764) Volume 2(2), March 2013. www.ijcit.com

[7] Garcia. D, Archer T, Moradi S, and Ghiabi B. (2012), Waiting in Vain: Managing Time and Customer Satisfaction at Call Centers. Science Research, http://dx.doi.org/10.4236/psych.2012.32030.

Psychology 2012. Vol.3, No.2, 213-216 Published Online February 2012 in SciRes http://www.SciRP.org/journal/psych)
[8] Hart M, Fichtner B, Fjalestad E, and Langley S. (2006) Contact centre performance: In pursuit of first call resolution. Management Dynamics, 15(4):17-28

[9] Jouini.O, Dallery .Y and Aksin .Z (2009). Queueing Models for MultiClass Call Centers with Real-Time Anticipated Delays. International Journal of Production Economics, 120:389-399.

[10] Mehrotra V, Ross K, Ryder G and Zhou Y (2012), Routing to Manage Resolution and Waiting Time in Call Centers with Heterogeneous Servers. Manufacturing \& Service Operations Management Vol. 14, No. 1, Winter 2012, pp. 66-81 ISSN 1523-4614 (print), $\quad$ ISSN $1526-5498 \quad$ (online) http://dx.doi.org/10.1287/msom.1110.0349 @2012

[11] Mehrotra .V, Ross .K, Ryder .G and Zhou .Y (2009), Routing to Manage Resolution and Waiting Time in Call Centers with Heterogeneous Servers. Manufacturing \& Service Operations Management 9(4): 167-181, ISSN 1523-4614 j EISSN 1526-5498

[12] Mughele, E.S. and Chiemeke S.C. (2016). A Performance Evaluation of Call Resolution Oriented Routing Rules to Enhance Resolution Rates. International Journal of Computer Application, Foundation of Computer Science, USA (0975 - 8887). Volume 143, No.8, available online at http://www.ijcaonline.org/

[13] Mughele E.S, Chiemeke C.S. and Konyeha .S (2017a), A Comparative Analysis of Waiting Time Routing Rule for Queue Reduction in Call Center. Journal Digital Innovations \& Contemporary Research in Science, Engineering \& Technology. Available online at http://www.isteams.net/digital-innovations-journal. Volume 5. N0 2

[14] Mughele E.S, Chiemeke C.S, Konyeha S., Ukaoha K.C and Akpon-Ebiyomare D. (2017b), Hybridized Optimisation Framework for Routing calls in Call Centres. FTC-2017, Future Technologies Conference, Vancouver, Canada 28-29 November 2017. Conference proceeding in IEEE Xpliore.

[15] Mughele E.S and Chiemeke C.S (2017) A Framework for the Optimisation of Routing Techniques in Call Centres" International Conference on Science, Technology, Education, Arts, Management and Social Science (iSTEAMS). Conference proceedings Pp. 413 424. Caleb University Imota Lagos State 29th May to $2^{\text {nd }}$ June 2017.

[16] Mughele, E.S, Chiemeke C.S and Chete C.F (2017c), "Simulation Model for Optimization of Call Center" AIMS Research Journal. Available online at http://www.isteams.net/aims-journal Volume 3 N0 2

[17] Mughele E. S, Chiemeke S. C and Ukaoha, K. C. (2017d), Call Center Optimisation Using Graph Theory Analysis, IEEE NIGERCON 2017, Federal University Of Technology Owerri, Imo State Nigeria $7^{\text {th }}$ to $10^{\text {th }}$ November 2017, Publication in conference proceeding in IEEE Xplore.

[18] Osahenvemwen . O and Odiase .O, (2016), Effective utilization of mobile call center using queuing models. International Journal of Engineering and Technology, Vol, 8, N0 2 (IACSIT) 
[19] Selvi .V and Sathya .P (2012) Mathematical Applications of Queuing Theory in Traffic Congestion. International Journal of Scientific \& Engineering Research, Volume 3, Issue 11, November -2012 12 ISSN 2229-5518

[20] Stanley .J, Saltzman R and Mehrotra V (2008), Improving call center operations using performancebased routing strategies. CJOM, 6(1): 24-32.

[21] V'ericourt P and Zhou Y (2005b). Managing Response Time in a Call-Routing Problem with Service Failure. OPERATIONS RESEARCH INFORMS Vol. 53, No. 6 ,
November-December 2005, pp. 268-281 ISSN 0030364X_ ISSN 1526-5463 _ 05 _ 5306 _0968

[22] Véricourt F and Zhou P (2005a). A routing problem for call centers with customer callbacks after service failure. Operations. Research. 53(6) 968-981.

[23] Zhan D and Ward A (2006) Threshold Routing to Trade-off Waiting and Call. Resolution in Call Centers MANUFACTURING \& SERVICE OPERATIONS MANAGEMENT Vol. 12, No. 2, pp. 316-323. ISSN 1523-4614 EISSN 1526- www bcf.usc.edu/ amyward/ZhWa_7_24_2013MSOM_Body. pd (Accessed June 2019) 